TITLE:

\title{
Molecular dynamics simulations study of nano particle migration by cluster impact
}

$\operatorname{AUTHOR}(\mathrm{S}):$

Aoki, Takaaki; Seki, Toshio; Matsuo, Jiro

\section{CITATION:}

Aoki, Takaaki ...[et al]. Molecular dynamics simulations study of nano particle migration by cluster impact. Surface and Coatings Technology 2016, 360(Part A): 63-68

\section{ISSUE DATE:}

2016-11-25

URL:

http://hdl.handle.net/2433/217223

\section{RIGHT:}

(c) 2016. This manuscript version is made available under the CC-BY-NC-ND 4.0 license

http://creativecommons.org/licenses/by-nc-nd/4.0/; The full-text file will be made open to the public on 25 November 2018 in accordance with publisher's 'Terms and Conditions for Self-Archiving'.; この論文は出版社版でありません。引 用の際には出版社版をご確認ご利用ください。; This is not the published version. Please cite only the published version. 
Highlights

- MD simulations were performed to explore the utility of gas cluster ion beam for cleaning nano particle (NP) on surface.

- MD result suggests the kinetic energy density of incident atoms may excess that of initial state and work to sweep NP.

- The study summarizes optimized cluster size and offset distance from NP for maximum migration of NP. 


\title{
Molecular Dynamics Simulations Study of Nano Particle Migration by Cluster Impact
}

Takaaki Aoki $^{\mathrm{a},}{ }^{*}$, Toshio Seki $^{\mathrm{b}}$, Jiro Matsuo ${ }^{\mathrm{c}}$

${ }^{a}$ Department of Electronic Science and Engineering, Graduate School of Engineering, Kyoto University, Nishikyo, Kyoto, 615-8510, JAPAN

b Department of Nuclear Engineering, Graduate School of Engineering, Kyoto University, Gokasho, Uji, 611-0011, JAPAN

c Quantum Science and Engineering Center, Graduate School of Engineering, Kyoto University, Gokasho, Uji, 611-0011, JAPAN

* Corresponding Author: E-mail: aoki.takaaki.6v@kyoto-u.ac.jp; Tel: +81-75-383-7136; Fax +81-75-383-7366; Postal Address: Department of Electronic Science and Engineering, Graduate School of Engineering, Kyoto University, Nishikyo, Kyoto, 615-8510, JAPAN

\begin{abstract}
Molecular dynamics (MD) simulations are performed in order to investigate the radiation effects of a huge and slow gas cluster for the surface cleaning process. When a large argon cluster with the size ranging from 20000 to 300000 is accelerated with a total of $30 \mathrm{keV}$, each constituent atom carries very low energy ranging from $1.5 \mathrm{eV} /$ atom to $0.1 \mathrm{eV} /$ atom. In many cases, the cluster does not penetrate the solid target surface but is deflected in a lateral direction. This collisional process results in a high density particle flow spreading along the surface plane due to cohesion of the cluster, which suggests the capability to modify the irregular surface structure, without damage in the target. The MD simulations demonstrate that such a huge cluster sweeps a nano particle (NP, $3 \mathrm{~nm}$ in radius) attached on a planar silicon target's surface. From
\end{abstract}


the investigation of various conditions of cluster impact, it is found that the migration distance is correlated with the kinetic energy applied on the NP by the impact of cluster atoms. Additionally, the MD results suggest the existence of optimized parameters for the maximum migration distance for the offset distance between the cluster and the NP, and the cluster size for constant total energy (equivalent to energy per atom or kinetic energy density). The optimized offset distance was estimated as the summation of radii of the incident cluster and the NP. The optimized energy per atom was suggested around $0.6 \mathrm{eV} /$ atom, where the cluster efficiently spreads in lateral direction keeping higher kinetic energy density of particle flow.

Keywords: Molecular dynamics simulation, Gas cluster ion, Surface cleaning

\section{Abstract code: P6}

\section{Introduction}

The gas cluster ion beam (GCIB) is a unique ion beam technique where a gas cluster, a large aggregation of source atoms/molecules, is generated as a cluster, and then ionized, accelerated, and radiated on the target. Several experiments [1, 2] have demonstrated that the glancing angle irradiation of an energetic cluster beam can remove surface roughness without damage, which suggests that the GCIB process can be applied for an efficient surface cleaning process under dry process conditions. The glancing angle incidence of the GCIB is a useful technique to treat a planar surface or the side wall of a trench and pillar structure. On the other hand, the demand for efficient surface cleaning with a dry process arises from the cleaning of semiconductor wafers with a mask structure kept on the surface. Dobashi [3] and his group reported that ultrafine 
particles are removed without damage on the wafer surface or pattern structure by utilizing a $\mathrm{CO}_{2} \mathrm{GCIB}$ at several tens of $\mathrm{keV}$ of incident energy.

In the previous works performed in both experiments $[4,5]$ and simulations [6], it has been demonstrated that low-damage cluster irradiation can be realized by controlling not only the incident energy but also the cluster size or incident angle. The value of the normal component of the incident energy per atom is proposed as a characteristic parameter to determine whether the incident cluster penetrates the target surface to cause crater-like damage. As mentioned before, a large cluster should be selected to reduce the damage under normal conditions.

For the cluster ion beam process, the incident energy per atom is an important parameter to cause surface damage, and it can be controlled by changing the cluster size. For example, when the total incident energy is $10 \mathrm{keV}$, the $\mathrm{X}_{1000}$ and $\mathrm{X}_{10000}$ clusters carry $10 \mathrm{eV} /$ atom and $1 \mathrm{eV} /$ atom, respectively. However, it is not clear whether such low-damage irradiation conditions have enough capability to remove surface contamination. In this paper, we demonstrate several molecular dynamics simulations of large and slow cluster impact on a solid target with solid nano particles attached. The motion and energy transfer process were investigated and the mechanisms of surface cleaning were discussed.

\section{Simulation Method and Model}

Molecular dynamics (MD) simulations were performed in order to examine the collisional process of a large cluster impacting on a solid surface and its cleaning effect. In this simulation, three different types of atom are prepared to represent an incident Ar cluster, $\mathrm{Si}(100)$ target and a nano particle. The interaction between the Si atoms is described by the Stillinger-Weber model 
in [7]. The other pairs, $\mathrm{Ar}-\{\mathrm{Ar}, \mathrm{Si}, \mathrm{NP}\}, \mathrm{NP}-\{\mathrm{Si}, \mathrm{NP}\}$, are governed by the Lennard-Jones 12-6 model described in Table 1 , in which the parameters are taken from $[8,9]$.

Additionally, the mass of the NP atom is defined as $12 \mathrm{amu}$. From these definitions, the bulk properties of the NP are

- density: $1.5 \mathrm{~g} / \mathrm{cm}^{3}$

- structure: fcc

- Young’s Modulus: 260 GPa.

These properties imply that the NP is hard enough so that it does not collapse as a result of the cluster impact with all conditions in this study. This fact may simplify the problem to discuss the dynamics among cluster, surface and NP. However, on the other hand, it is noted that the parameter set in table 1 is artificially designed by the authors so it does not match with real materials. The NP consists of 10000 NP atoms, and has a spherical structure with 3 nm radius. After the preliminary simulation of the structural optimization of the NP attached on the $\operatorname{Si}(100)$ surface, the binding energy between the NP and surface is calculated as about $70 \mathrm{eV}$.

The target $\mathrm{Si}(100)$ surface includes more than 2 million atoms and has $69.5 \times 69.5 \mathrm{~nm}$ of surface area and $8.6 \mathrm{~nm}$ of depth. The atoms in the region 1 unit cell length forming the edge and bottom of the target are fixed to keep a diamond structure, and several layers inside it act as a thermal bath by Langevin dynamics to absorb excess impact energy and keep the target temperature at $300 \mathrm{~K}$. Various sizes of Ar clusters from 20000 to 300000 were prepared as the projectiles. In this work, the total kinetic energy of projectile is fixed as $30 \mathrm{keV}$, so the incident energy per atom of projectiles varies from $1.5 \mathrm{eV} /$ atom to $0.1 \mathrm{eV} /$ atom. These projectiles were radiated on the target along the surface normal, aimed at several points on the target away from the NP center. The incident angle for the projectile to impact on surface was not varied. 


\section{Results and discussion}

Firstly, the dynamics of incident cluster atoms to a planar target surface is examined. Fig. 1 shows the map particle and kinetic energy density of the argon atoms during the impact of $\operatorname{Ar}_{100000} 30 \mathrm{keV}$ on a planar $\mathrm{Si}(100)$ target. The size of the mesh point is $0.5 \mathrm{~nm} \times 0.5 \mathrm{~nm}$.

When the incident cluster makes contact with the target surface, the bottom of the cluster is compressed and the particle density increases at the impact center. On the other hand, the cluster atoms around the impact point do not move anymore or penetrate the target surface, so the kinetic energy density for these atoms decreases and the kinetic energy is transferred to the edge of the contact region, where the atoms can move outside (Figs. 1(b) and 1(c)). Through the multiple collisions inside the cluster, the initial momentum of the incident cluster along the surface cluster is deflected to the parallel to the surface. As shown in Figs. 1(c) and 1(d), the most energetic part can be found at the edge of the cluster, and this area also moves outside, spreading and gradually decreasing in value. Meanwhile, the particle density map indicates that the densest area continues to reside around the impact center for long time, but the atoms in this area carry less kinetic energy.

The trajectory and time evolution of the most energetic point are shown in Fig. 2. Fig. 2(a) shows the trajectory of the mesh point in cylindrical coordinate ( $z$ : height, $r$ : radius) which gives the maximum kinetic energy density at each moment, whereas Fig. 2(b) represents the time and the value of the corresponding point in Fig. 2(a). From Fig. 2 it can be concluded that the highest energy density can be realized at $(t, r, z)=(10 \mathrm{ps}, 10.75 \mathrm{~nm}, 0.75 \mathrm{~nm})$ with the value of 18 $\mathrm{eV} / \mathrm{nm}^{3}$, as indicated by the arrow symbols. Considering the discussion in Fig. 1, it is supposed that this point is related with the radius of the incident cluster. Additionally, it is interesting that 
the value at the energetic point may surpass the initial kinetic energy density $\left(11 \mathrm{eV} / \mathrm{nm}^{3}\right)$, and this effect is expected to contribute to the surface smoothing and cleaning.

As the time proceeds, the energetic point moves outside and its value also diminishes. Fig. 2(a) shows that the most energetic mesh point jumps randomly between the outside and impact center after a long time has passed, which means that the characteristic collective motion due to the cluster impact does not occur.

Fig. 3 shows the MD simulation results of the Ar cluster impacting on the Si(100) target with the attached NP. The snapshots represent the cross-sectional view, which are cut along the center line of the cluster, the target and the NP. The incident cluster consists of 100,000 atoms and is accelerated with a total of $30 \mathrm{keV}$ (namely $0.3 \mathrm{eV} /$ atom). For all conditions, the clusters are radiated along the surface normal of the Si targets, with a varying offset distance from the NP. The offset distance is chosen between 0 and $24 \mathrm{~nm}$. Here, it is noted that the radii of the incident cluster and NP are about $9.5 \mathrm{~nm}$ and $3 \mathrm{~nm}$, respectively. Additionally, the time transition of the position and the kinetic energy of the NP are shown in Fig. 4. Fig. 4(a) shows the trajectories of the center of mass of the NPs. The circles in the figure indicate the final position of the NPs at 77 ps, which are equivalent to the bottom snapshots in Fig. 3. On the other hand, Fig. 4(b) represents the time transition of the kinetic energy of NPs.

Figs. 3 and 4 suggest that the motion of the NP differs according to the offset distance. Identically, when the offset distance is 0 , the incident cluster atoms hit the both sides of the NP symmetry. Thus, the lateral migration of NP is suppressed and the NP moves only along the surface normal. In this case, from the detailed motion shown as blue line and circle in Fig. 4, the center of mass of the NP first moves downward to reach $2.5 \mathrm{~nm}$ from the surface, and is then reflected and remains $2.8 \mathrm{~nm}$, which corresponds that the bottom edge of the NP (with $3 \mathrm{~nm}$ 
radius) moves once to $0.5 \mathrm{~nm}$ and remains at $0.2 \mathrm{~nm}$ under the surface level, respectively. Additionally, it can be found in Fig. 3(a) that the target surface atoms around the contact point are disordered, which means that damage may be caused with a specific collisional process.

In Figs. 3(b), 3(c) and 3(d), the flow of cluster atoms becomes asymmetric and hits the NP on one side, which contributes to the NP motion in a lateral direction. When the offset distance is 6 nm (Fig. 3(b)), a part of the incident cluster atom collides with the NP directly. The NP gains large kinetic energy and moves rapidly. In this case, it is noted that the NP moves to more than $34 \mathrm{~nm}$ after $60 \mathrm{ps}$, which means that the NP is out of range of the target area, making it difficult to discuss the migration distance and energy profile.

For the larger offset distance (cases of Fig. 3(c) and 3(d)), most of the cluster atoms collide with Si target atoms first, rather than NP atoms, and are then deflected in parallel to the surface plane. Even in such case, the collective flow of deflected atoms shows the capability to apply enough kinetic energy for NP migration. The kinetic energy transfer diminishes as the offset distance increases.

From Fig. 4(b), when the offset distance is 6, 12 or $18 \mathrm{~nm}$, the kinetic energy of the NP rises rapidly and then decreases gradually. This means that the NP gains a large amount of kinetic energy by the impact-like collision and loses it by friction with the target surface. Additionally, it is suggested that there seems to be a threshold energy where the NP starts to migrate and that it is related with the binding energy between the target and the NP.

For further discussion, the relationships among the impact offset distance, maximum gain of NP kinetic energy and migration distance on the $\mathrm{x}$-axis for various offset distances are shown in Fig. 5. From Fig. 5(c), it is suggested that the migration distance is generally correlated with the maximum kinetic energy gain. However, if one looks carefully, when the offset distance is small, 
the migration distance is small, even if the same kinetic energy is given. This seems to be due to the implantation of the NP or the effect that some cluster atoms override the NP, as shown in Figs 3(a) and 3(b). In this simulation condition, the highest kinetic energy gain is observed around $7.5 \mathrm{~nm}$ of offset distance, and these offset distance values lead to a larger NP migration, as in Fig. 5(b). This result agrees well with the results and discussion in Figs. 1 and 2.

Fig. 6 is the same as Fig. 5, but includes the results for different cluster sizes ranging from $\mathrm{Ar}_{20000}$ to $\mathrm{Ar} 300000$. The total incident energy is constant at $30 \mathrm{keV}$, but the incident energy per atom and the cluster radius are different. For example, $\mathrm{Ar}_{20000}$ has a $5.45 \mathrm{~nm}$ radius and carries $1.5 \mathrm{eV} /$ atom, while $\mathrm{Ar}_{300000}$ has $13.6 \mathrm{~nm}$ and $0.1 \mathrm{eV} /$ atom. As shown in Fig. 6(c), when the offset distance is large enough, the offset distance and migration distance show good correlation. However, explicit divergence from the correlation can be found when both the cluster size and offset distance are small.

From Figs. 6(a) and (b), it is supposed that the optimized offset distance which gives the maximum kinetic energy and migration distance depends on the cluster size and is related with the radius of the incident cluster. Moreover, there is an optimized condition of Ar50000 (0.6 eV/atom) to realize the maximum migration distance. If the cluster size is as small as 20000 and the incident energy per atom is as large as $1.5 \mathrm{eV} /$ atom, the NP penetrates deeper than in other conditions because of the larger energy transform by the direct impact at a small offset distance, which may interfere with the lateral migration. Additionally, when the offset distance is larger than $10 \mathrm{~nm}$ (which corresponds to the sum of the radii of the cluster and NP), the incident cluster impinges itself to create a small dimple on the silicon target surface. After impinging, the cluster atoms are reflected from the target. The direction of the reflected atoms contains a vertical 
component and does not tend to flow in parallel to the surface. This motion of incident atoms results in the reduction of kinetic energy transfer to the NP.

On the other hand, if the total incident energy is constant, increasing the cluster size means decreasing the incident energy per atom as well as the kinetic energy density. When the offset distance is small and the cluster and NP collide directly, the kinetic energy gain of the NP by impact is simply estimated by multiplying the kinetic energy density and the area of the NP. This means that the kinetic energy gain is reduced as the cluster size increases. When the offset distance is large enough, it is estimated that the cluster atoms included in the projection area from the NP to the cluster contribute to the motion of the NP. Under this estimation, the sum of the kinetic energy of the atoms to be projected to the NP is constant. This may be one of the reasons that the kinetic energy gain and migration distance become universal for various cluster sizes.

\section{Conclusion}

The impact of a huge and slow cluster on a solid target has been investigated by molecular dynamics (MD) simulation from the viewpoint of surface cleaning. By utilizing a huge cluster such as $\mathrm{Ar}_{100000}$, a very slow but high density particle flux can be realized. The initial stage of the impact between a cluster and a solid target is mainly dominated by the incident energy per atom rather than the total kinetic energy. Therefore, if the incident energy per atom or the incident energy density is less than the surface binding energy, the incident cluster atoms cannot penetrate the target surface, but are compressed at the interface. As a result of the multiple collision effect at the interface, the cluster atoms spread in parallel to the surface as a corrective flow of dense 
energetic particles, which may contribute to the characteristic surface modification process, such as nano particle (NP) migration.

The MD results of cluster impacts with various parameters revealed that the NP starts to move when it gains sufficiently large kinetic energy from a collision impact to overcome the binding energy with the target. After migration, the NP loses its kinetic energy and stops by friction with the target. This kinetics means that the kinetic energy gain and migration distance have good correlation with each other. The investigation of the impact offset's dependence on the kinetic energy gain (and migration distance) showed that there is an optimized offset distance to cause the longest migration distance, and it is nearly equal to the summation of the radii of the cluster and the NP. These results are also explained by the investigation that the point of highest energy density can be realized at the edge of the incident cluster in the radial axis and at the near surface level. Additionally, the magnitude of the kinetic energy density may exceed that which the cluster carries initially.

In this paper, the dynamics of the cluster impact were described in only qualitative terms. Formulation of the migration process depending on the cluster and NP size, the incident cluster energy and the binding energy of the NP will be studied by further MD simulation and detailed discussion.

\section{References}

[1] E. Bourelle, A. Suzuki, A. Sato, T. Seki, J. Matsuo, Sidewall polishing with a gas cluster ion beam for photonic device applications, Nucl. Instruments Methods Phys. Res. Sect. B Beam Interact. with Mater. Atoms 241 (2005) 622-625. 
[2] S. Kakuta, S. Sakaki, T. Hirano, K. Ueda, T. Seki, S. Ninomiya, et al., Low damage smoothing of magnetic material films using a gas cluster ion beam, Nucl. Instruments Methods Phys. Res. Sect. B Beam Interact. with Mater. Atoms 257 (2007) 677-682.

[3] K. Dobashi, K. Inai, M. Saito, T. Seki, T. Aoki, J. Matsuo, Ultrafine particle removal using gas cluster ion beam technology, IEEE Trans. Semicond. Manuf. 26 (2013) 328-334.

[4] N. Toyoda, S. Houzumi, I. Yamada, Development of a size-selected gas cluster ion beam system for low-damage processing, Nucl. Instruments Methods Phys. Res. Sect. B Beam Interact. with Mater. Atoms 241 (2005) 609-613.

[5] T. Seki, T. Murase, J. Matsuo, Cluster size dependence of sputtering yield by cluster ion beam irradiation, Nucl. Instruments Methods Phys. Res. Sect. B Beam Interact. with Mater. Atoms 242 (2006) 179-181.

[6] T. Aoki, J. Matsuo, Molecular dynamics simulations of surface modification and damage formation by gas cluster ion impacts, Nucl. Instruments Methods Phys. Res. Sect. B Beam Interact. with Mater. Atoms 242 (2006) 517-519.

[7] F. Stillinger, T. Weber, Computer simulation of local order in condensed phases of silicon, Phys. Rev. B 31 (1985) 5262-5271.

[8] A. Rahman, Correlations in the motion of atoms in liquid argon, Phys. Rev. 136 (1964) A405-A411.

[9] S. Satake, Large-scale molecular dynamics simulation for two Ar clusters impact on 4H-SiC, Nucl. Instruments Methods Phys. Res. Sect. B Beam Interact. with Mater. Atoms 257 (2007) 639-644.

\section{Tables}


Table 1: Potential models and parameters for MD simulations in this study.

\begin{tabular}{|l|l|l|l|}
\hline Atomic Pairs & $\varepsilon[\mathrm{eV}]$ & $\sigma[\mathrm{nm}]$ & Reference, note \\
\hline Ar-Ar & 0.01034 & 0.34 & {$[7]$} \\
\hline Ar-Si & 0.005 & 0.375 & {$[8]$} \\
\hline Ar-NP & 0.005 & 0.375 & Same as Ar-Si \\
\hline NP-NP & 0.5 & 0.237 & Given by authors \\
\hline NP-Si & 0.2 & 0.20951 & $\varepsilon$ is given by authors \\
& & & $\sigma$ is same as Si-Si [6] \\
\hline
\end{tabular}

\section{Figure Captions}

Fig 1: Particle and kinetic energy density mapping of the incident argon atoms at the impact of $\operatorname{Ar}_{100000} 30 \mathrm{keV}$ cluster on planar Si(100) target.

Fig 2: Trajectory of the mesh point which gives the maximum kinetic energy density at each moment (a) and time transition of the value at the point (b). In (a), the radius of incident cluster is superimposed. The arrow symbol indicates the position and time where the maximum kinetic energy density was observed.

Fig 3: Snapshots of $\mathrm{Ar}_{100000} 30 \mathrm{keV}$ impact on $\mathrm{Si}(100)$ target with nano particle attached on the target.

Fig 4: Trajectories of center of mass (a) and kinetic energy transitions (b) of the nano particle irradiated with $\mathrm{Ar}_{100000} 30 \mathrm{keV}$ cluster with different impact offset. 
Fig. 5: Relationship among impact offset, maximum gain of kinetic energy and final migration distance of the nano particle. The numbers in (c) represent the impact offset distances.

Fig. 6: Relationships among impact offset, maximum gain of kinetic energy and final migration distance of the nano particle for various cluster sizes ranging from $\mathrm{Ar}_{20000}(1.5 \mathrm{eV} / \mathrm{atom})$ to $\operatorname{Ar} 300000$ (0.1 eV/atom). 
(a) $t=0$
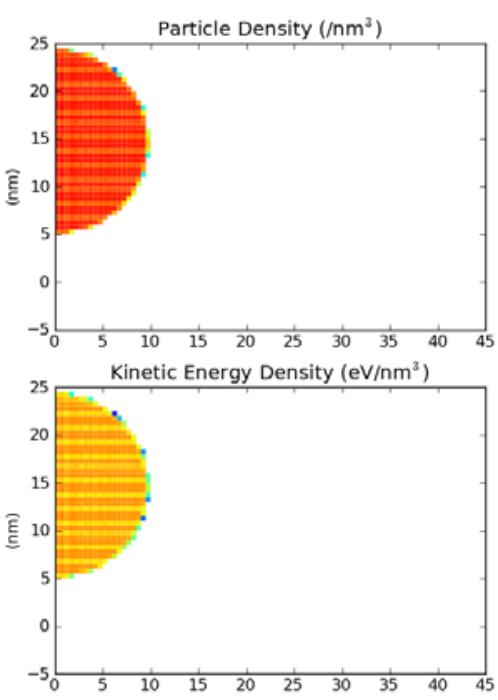

(d) $t=23.1 \mathrm{ps}$
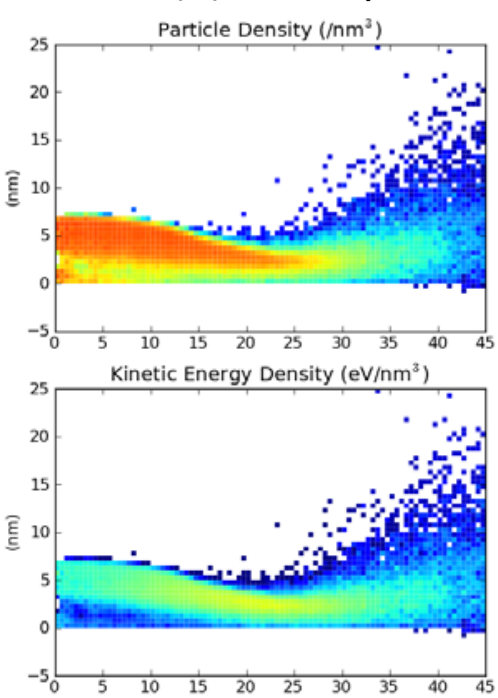

(b) $t=7.7 p s$
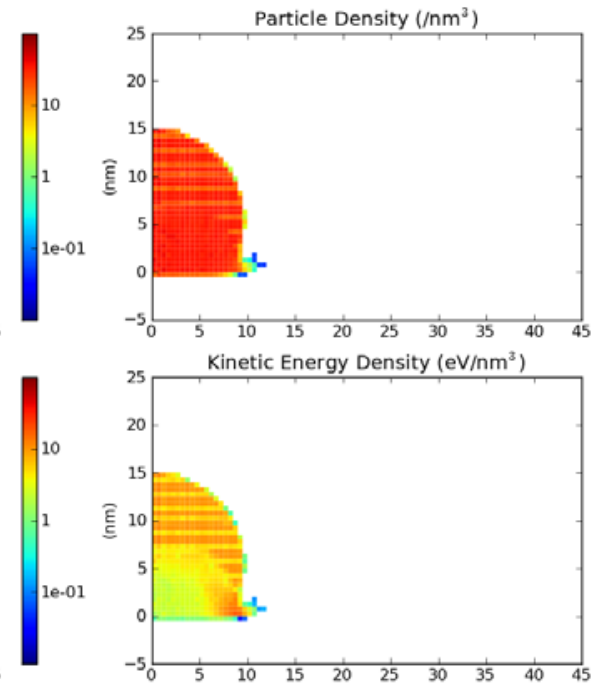

(e) $t=38.5 p s$
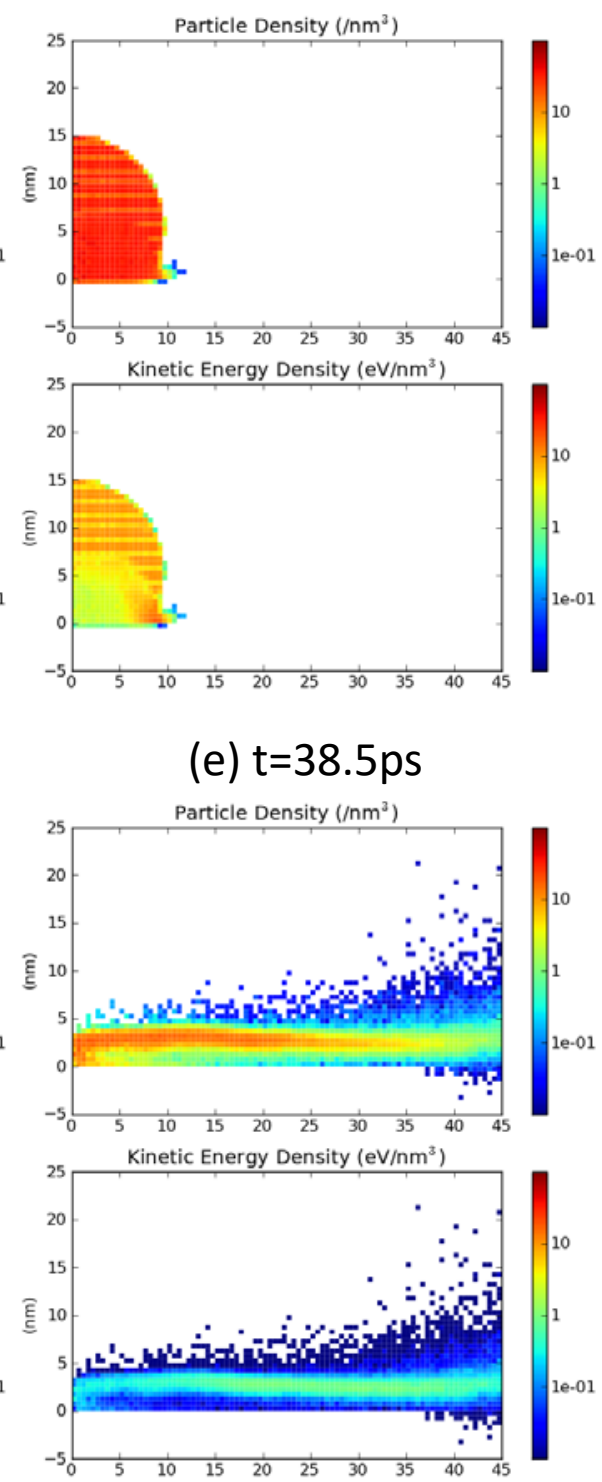

(c) $t=15.4 p s$
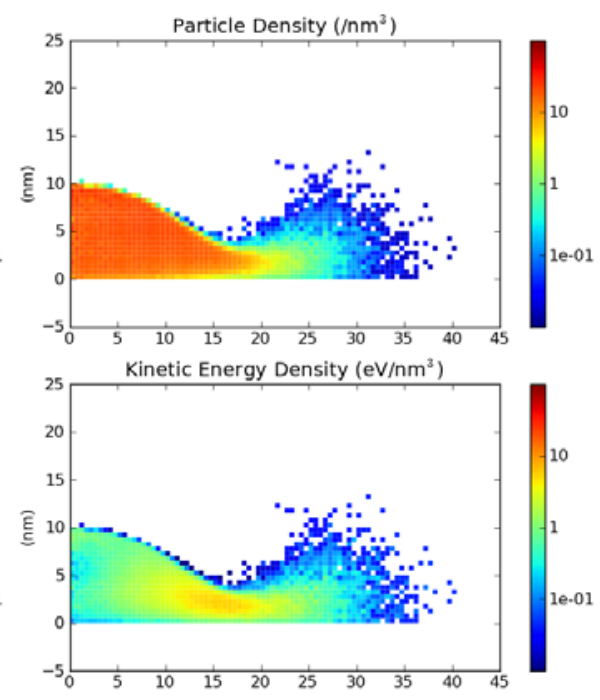

(f) $t=77 p s$
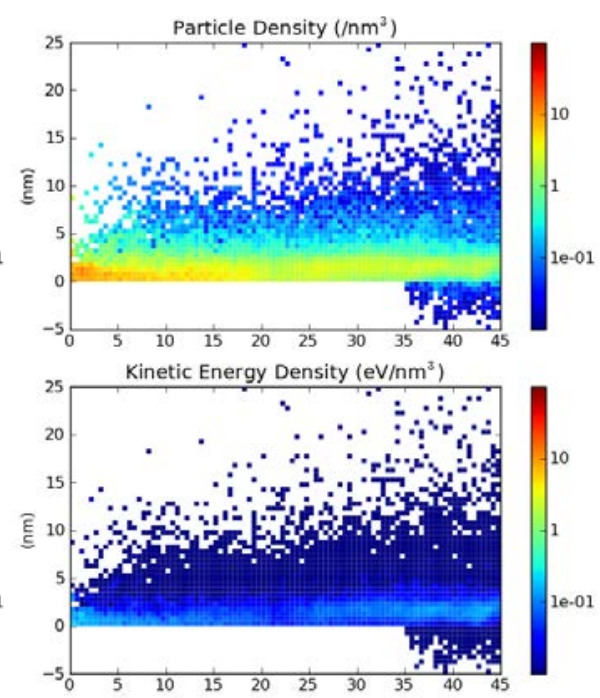

Fig 1: Particle and kinetic energy density mapping of the incident argon atoms at the impact of $\mathrm{Ar}_{100000} 30 \mathrm{keV}$ cluster on planar Si(100) target. 

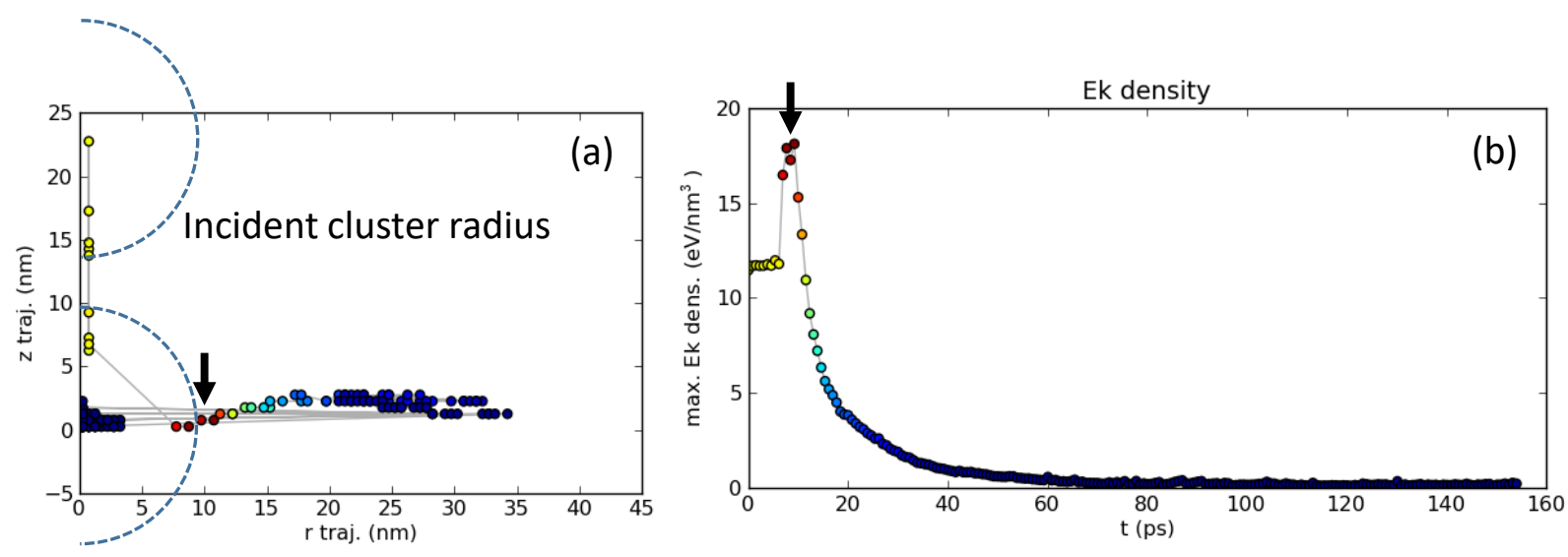

Fig 2: Trajectory of the mesh point which gives the maximum kinetic energy density at each moment (a) and time transition of the value at the point (b). In (a), the radius of incident cluster is superimposed. The arrow symbol indicates the position and time where the maximum kinetic energy density was observed. 


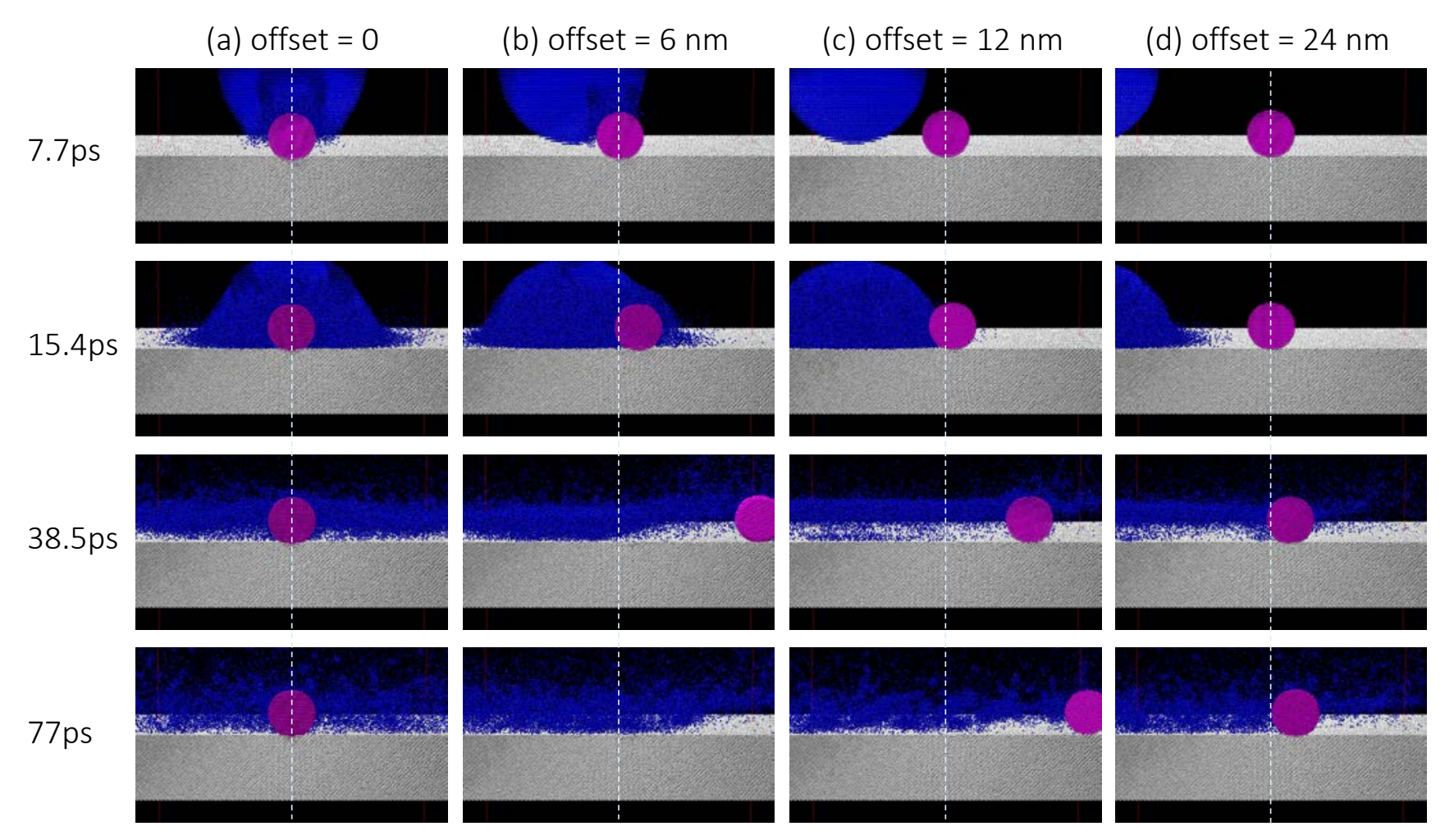

Fig 3: Snapshots of $\mathrm{Ar}_{100000} 30 \mathrm{keV}$ impact on $\mathrm{Si}(100)$ target with nano particle attached on the target. 

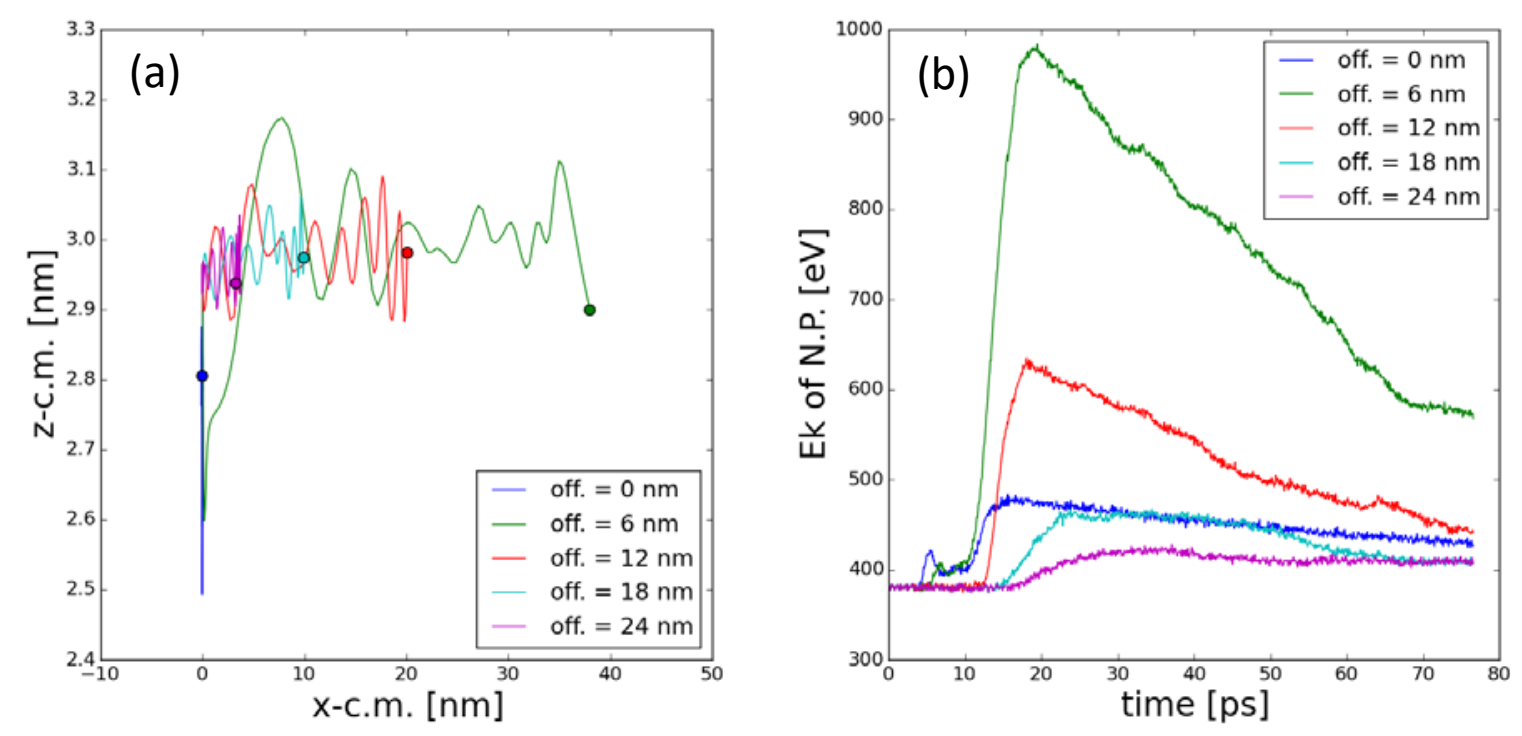

Fig 4: Trajectories of center of mass (a) and kinetic energy transitions (b) of the nano particle irradiated with $\mathrm{Ar}_{100000} 30$ keV cluster with different impact offset. 
(a)

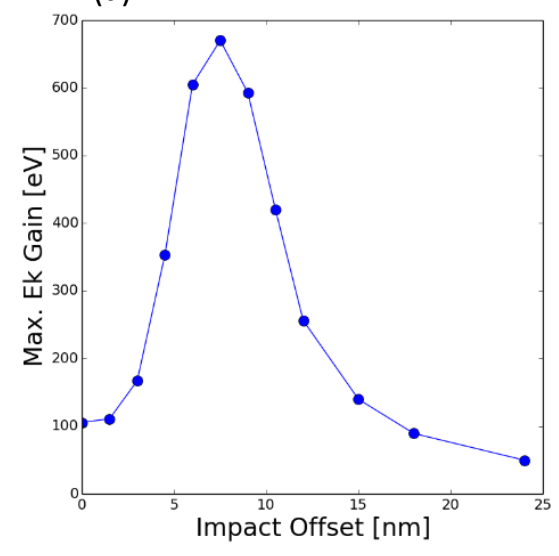

(b)

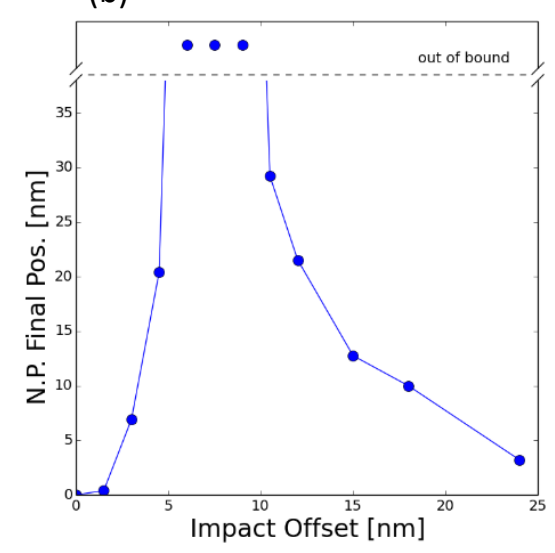

(c)

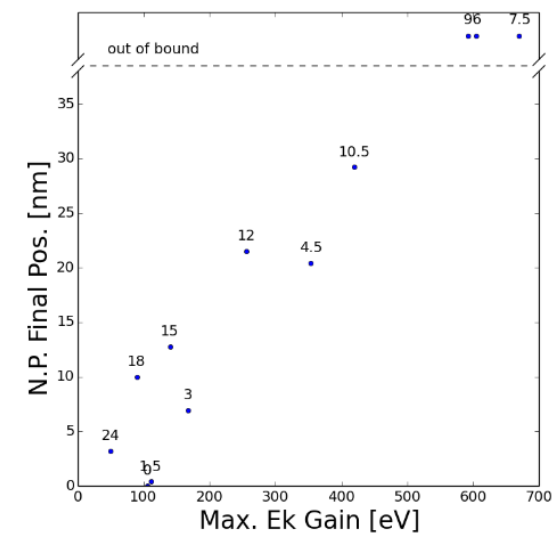

Fig. 5: Relationship among impact offset, maximum gain of kinetic energy and final migration distance of the nano particle. The numbers in (c) represent the impact offset distances. 
(a)

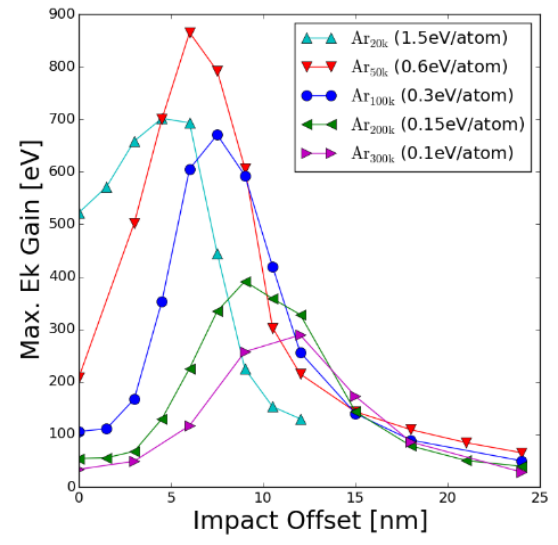

(b)

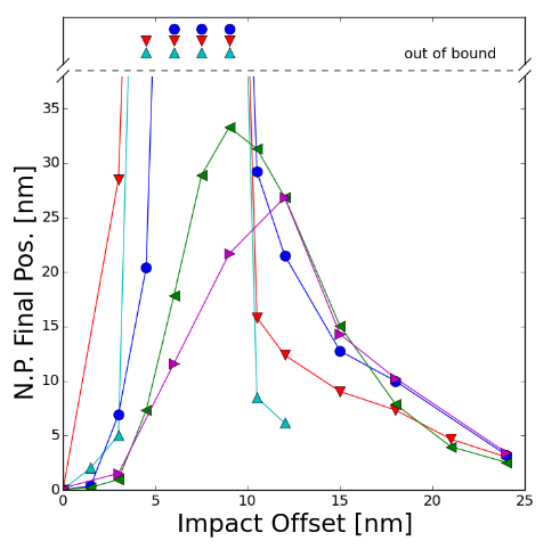

(c)

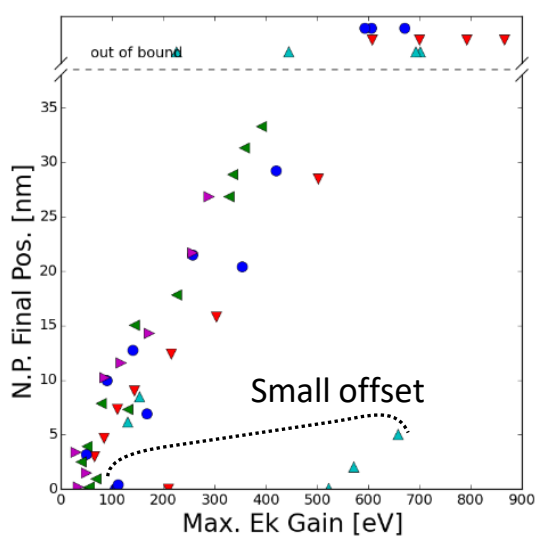

Fig. 6: Relationship among impact offset, maximum gain of kinetic energy and final migration distance of the nano particle for various cluster sizes ranging from $\operatorname{Ar}_{20000}$ (1.5 eV/atom) to $\mathrm{Ar}_{300000}$ (0.1 eV/atom). 